An vier Patienten erfolgreich

\title{
Immunglobulin stoppt Alzheimer!
}

Immunglobuline können eine Alzheimer-Demenz aufhalten, so das Ergebnis einer aktuellen Studie.

24 Patienten mit leichter bis moderater Alzheimererkrankung bekamen sechs Monate lang Immunglobuline oder Placebo. Je vier Patienten erhielten entweder $0,2 \mathrm{~g} \mathrm{IVIG} / \mathrm{kg}$ alle zwei Wochen, $0,4 \mathrm{~g} / \mathrm{kg}$ alle zwei oder vier Wochen oder $0,8 \mathrm{~g} / \mathrm{kg}$ alle vier Wochen. Die übrigen acht bekamen Placebo. Nach sechs Monaten ergab sich ein Vorteil für die Immuntherapie beim ADAS-cog-Wert sowie beim klinischen Gesamteindruck, hieß es auf der Alzheimer's Association International Conference in Toronto. Anschließend wurde die Studie offen ein Jahr fortgeführt, wobei alle Patienten IG in unter- schiedlichen Dosierungen bekamen. Schließlich wurde den Patienten nochmals eine Verlängerung um 18 Monate angeboten, mit der einheitlichen Dosis von $0,4 \mathrm{~g} / \mathrm{kg}$ alle zwei Wochen, da diese die beste Wirksamkeit zeigte.

\section{Kognitionsabbau gebremst}

Bei den vier Patienten, die über drei Jahre hinweg $0,4 \mathrm{~g} / \mathrm{kg}$ alle zwei Wochen erhalten hatten, konnte der Verlauf der Erkrankung gestoppt werden: Sowohl auf verschiedenen Kognitionsskalen, beim klinischen Gesamteindruck als auch bei den neuropsychiatrischen Symptomen verschlechterten sich die Werte nicht.

(mut)

Relkin N et al, AAIC 2012, Abstract P3-381

\section{Erratum zu:}

\section{Aktuelles in puncto Blutverdünnung}

Antikoagulanzien bei kardiovaskulären Erkrankungen

Dr. Thomas Höchtl, Dr. Serdar Farhan, Prof. Dr. Kurt Huber

3. Medizinische Abteilung mit Kardiologie und Internistischer Notaufnahme, Wilhelminenspital, Wien

CME 2012 - 9(5): 7-15 DOI 10.1007/s11298012-1238-6 • ( ) Springer-Verlag 2012

\section{Erratum}

Im Abschnitt „Neue Antithrombine bei nichtvalvulärem Vorhofflimmern" (S. 13 im Beitrag) wurde fälschlicherweise behauptet, dass der Wirkstoff Apixaban im November 2011 in den USA für die Prävention von Schlaganfällen und systemischen Embolien bei nichtvalvulärem Vorhofflimmern zugelassen wurde. Apixaban war zum Zeitpunkt der Veröffentlichung hierfür noch nicht zugelassen. Im November 2011 wurde in den USA die Zulassung beantragt. Eine Entscheidung hierzu wird für Ende Juni 2012 erwartet. In Europa wird der Zulassungsantrag für Apixaban zur Zeit durch die EMA bearbeitet, so der Hersteller.

Im gleichen Abschnitt wurde geschrieben, dass zum Wirkstoff Apixaban ein Rote-Hand-Brief des Herstellers existiert - dieser Hinweis ist falsch. Der Hinweis wurde bei der Korrektur vom Lektorat falsch zugeordnet und bezieht sich korrekterweise auf den anschließenden Abschnitt zu Dabigatran (Rote-HandBrief Pradaxa ${ }^{\oplus}$, Boehringer Ingelheim; http://www.ifap.de/fileadmin/user_upload/AMTS-Nachrichten/20111027_RoteHand-Brief_Pradaxa.pdf).

Die Faktor Xa Inhibitoren wurden im Beitrag unter dem falschen Begriff „Antithrombine" subsumiert. Korrekterweise lautet der Überbegriff „Antithrombotika“.

Wir bitten, die Fehler zu entschuldigen.

Wissenschaftler, oder auch Additive wie Nitrit sowie die vielen gesättigten Fette in verarbeitetem Fleisch.

$(r b)$

CME 2012 - 9(7/8): 68 - DOI 10.1007/s11298-

012-1265-3 • ( ) Springer-Verlag 2012 